\title{
CONTAMINACIÓN FÍSICA Y MICROBIOLÓGICA DEL CHILE “CHIPOTLE” DURANTE EL DESHIDRATADO
}

\section{PHYSICAL AND MICROBIOLOGICAL CONTAMINATION OF THE SMOKED "CHIPOTLE" PEPPER DURING DEHYDRATION}

\author{
Graciela D. Ávila-Quezada*, Cinthia I. Islas-Valenzuela, Ezequiel Muñoz-Márquez y Esteban Sánchez- \\ Chávez
}

Centro de Investigación en Alimentación y Desarrollo A. C. Av. 4a Sur No. 3820. Fracc. Vencedores del Desierto. 33089, Delicias, Chihuahua. Tel. y Fax 01(639) 474-8704

* Autor para correspondencia (gavilaq@ciad.mx)

\section{RESUMEN}

Una alternativa de comercialización del chile 'Jalapeño' (Capsicum annum L.) en México es mediante el proceso del ahumado y deshidratado cuyo producto es conocido como "Chipotle". El ahumado es una técnica que además de conservar los alimentos les proporciona un aroma y sabor especial, y se realiza tradicionalmente en un horno a campo abierto, por lo que existe la preocupación de la posible contaminación fecal y física acarreada por el viento. En este trabajo se determinó la calidad del "Chipotle" en el eslabón de la cadena de producción: ahumado y deshidratado, en función de tratamientos asociados con buenas prácticas de manejo (BPM) para este proceso. Los tratamientos para reducir al mínimo el riesgo de contaminación durante el proceso fueron: 1 . Colocación de una malla antigranizo sobre el horno; 2. Evitar el contacto directo del obrero con el fruto; y 3. Desinfestar los instrumentos de trabajo. Con estas sencillas BPM se logró una buena calidad microbiológica (coliformes fecales y $E$. coli no detectables) y ausencia de contaminación física del "Chipotle". Cuando no se aplicaron todas las actividades relacionadas con las BPM se observó contaminación con coliformes fecales, hasta de $210 \mathrm{NMP} / \mathrm{g}$ en el fruto de "Chipotle".

Palabras clave: Capsicum annum, prácticas de manejo, inocuidad.

\section{SUMMARY}

One marketing alternative for 'Jalapeño' pepper (Capsicum annum L.) is the smoked and dried processing whose product is known in México as "Chipotle". Smoking is a common technique of food preservation that provides a special aroma and flavor, and is traditionally carried out in a field open-oven, so that fecal and physical contamination carried by the wind is possible. In this work the microbiological quality of "Chipotle" was determined in the production chain: smoking and dehydration, according to treatments for good manufacturing practices (GMP) in this process. The treatments to minimize the risk of contamination during the process were: 1. Placing a mesh hail (mesh-shade) over the drying oven; 2. Restricting direct contact of the worker with the chile pepper; and 3. Sanitizing working tools. With these simple GMP fruit quality and absence of physical contamination of "Chipotle" were attained. When not all GMP-related activities were implemented, contamination of the "Chipotle" with fecal coliforms reached values up to 210 MPN/g in the "Chipotle" fruits.

Index words: Capsicum annum, manufacturing practices, food safety.

\section{INTRODUCCIÓN}

En la cadena productiva del chile (Capsicum annum L.) "Chipotle" deshidratado, tanto el productor, el procesador como el comercializador deben tener conocimiento de los riesgos de contaminación física, química y microbiológica en cada una de las etapas de producción (Jiménez y Chew, 2002). El principio de las buenas prácticas lo constituye el conocimiento de que todo lo que entre en contacto físico con frutas y hortalizas puede causar contaminación, y que la mayoría de microorganismos patógenos y la contaminación física provienen del hombre y de los animales (Vlkova et al., 2006).

Los organismos coliformes totales y fecales son indicadores de contaminación en los alimentos (Leclercq et al., 2002; NMX-F-308-1992). Principalmente se encuentran en suelo, agua contaminada, materia en descomposición, animales, insectos e intestino grueso del hombre (Fairbrother y Nadeau, 2006; Vlkova et al., 2006). Los reglamentos internos propios de cada país indican un número máximo permisible de organismos coliformes totales y fecales en los alimentos, lo cual determina si es apto o no para su consumo. En México estos límites se indican en las Normas Oficiales Mexicanas y en las Normas Mexicanas de las Secretarias de Salud y de Agricultura (NOM-112-SSA1-1994; NMX-FF-108SCFI-2007). 
La bacteria Escherichia coli 0157:H7 (Ec0157) se identificó por primera vez en 1975 como posible patógeno de humanos en California, EE.UU., aunque el origen de la bacteria se asoció con animales silvestres, especialmente rumiantes (Doyle et al., 2006). En estos últimos años esta bacteria ha provocado muchos brotes de enfermedades gastrointestinales en el mundo (Hilborn et al., 1999; Pouch e Ito, 2001; Söderström et al., 2005). El límite de tolerancia para Ec0157 es cero, es decir, no debe estar presente en ningún alimento (NOM-145-SSA1-1995), por su alta virulencia. La Norma Mexicana indica que el chile "Chipotle" debe estar libre de E. coli (NMX-FF-108SCFI-2007).

Los sistemas de calidad pueden ayudar a reducir los riesgos de contaminación de los alimentos (Martínez et al., 2005). Las buenas prácticas agrícolas (BPA) y buenas prácticas de manejo (BPM) reducen los riesgos microbiológicos, físicos y químicos en la producción, cosecha y acondicionamiento en campo, procesamiento, empaque, transporte y almacenamiento. Además de los aspectos de higiene e inocuidad para alcanzar la sustentabilidad de la producción agrícola, se agrega la salud de los trabajadores y el cumplimiento de las normativas laborales dentro del marco de la producción agraria comercial (FAO, 2008).

En el caso particular de hortalizas que crecen cerca del suelo, como el chile, se ha visto la necesidad de aplicar BPA para garantizar que este producto no cause daños al consumidor. Se ha incrementado notablemente la producción y demanda del chile en México y en el mundo en los últimos años. En México la producción aumentó de chile de 668579 t en 1994 a 1989559 t en el 2007, y el Estado de Chihuahua es el segundo productor nacional con $564140 \mathrm{t}$ en 2007 (SIAP, 2008). Ante tal incremento en la producción se ha estado recurriendo a dar un valor agregado al producto, mediante un proceso de industrialización. El procesamiento del "Chipotle" involucra el ahumado y deshidratado con calor y humo de madera en un horno al aire libre. De aquí surge la interrogante sobre la calidad microbiológica del producto ya que se procesa durante $6 \mathrm{~d}$ con exposición al ambiente, donde corre el riesgo de contaminarse con materia fecal, principalmente.

Con la finalidad de llevar este producto a la industria con la mejor calidad, de acuerdo con la NMX-FF-108SCFI-2007, y debido a que la primera parte del proceso del "Chipotle" se hace al aire libre, el objetivo de este trabajo fue determinar los puntos de contaminación física y microbiológica, e identificar los posibles riesgos de contaminación en el proceso producción del "Chipotle" mediante la aplicación de BPM.

\section{MATERIALES Y MÉTODOS}

\section{Experimento 1, Horno chipotlero}

El experimento se estableció en un horno ubicado en el Parque Industrial Chipotlero en Camargo, Chihuahua, México, en septiembre del 2003. El horno fue construido previamente por el productor, con cuatro paredes de bloques de concreto de $1.30 \mathrm{~m}$ de altura, $4 \mathrm{~m}$ de longitud y $3 \mathrm{~m}$ de ancho. Sobre el horno se ubicaron rejillas de madera, espaciadas a $0.6 \mathrm{~cm}$ para permitir la salida del humo generado por la combustión de madera. Sobre las rejillas se colocó el chile fresco de color rojo proveniente del campo.

Tratamientos. Para diagnosticar los posibles puntos de contaminación se probaron cuatro tratamientos. El horno chipotlero se dividió en cuatro espacios de $1 \mathrm{~m}$ x $3 \mathrm{~m}$ cada uno, y en cada espacio se estableció un tratamiento. Los cuatro tratamientos se dividieron con hileras de bloques de concreto. El Tratamiento I (Cuadro 1) consistió en lavar la superficie del horno con agua y jabón, y luego se asperjó una solución clorada de $150 \mathrm{~mL} \mathrm{~L}{ }^{-2}$. En los Tratamientos II y III sólo se asperjó el horno con la solución clorada, y en el Tratamiento IV no se limpió, ni se desinfectó, ni se protegió. Después de aplicados los tratamientos se colocó la malla antigranizo para cubrir el chile de los Tratamientos I, II y III.

Inicio del ahumado. Se aplicó a $400 \mathrm{~kg}$ de chile 'Jalapeño' rojo provenientes de la parcela y que se depositaron en cada uno de los cuatro espacios (uno por tratamiento). El espesor de la capa de chile fue de aproximadamente $23 \mathrm{~cm}$. Se encendió la madera en la cavidad del horno para empezar el ahumado del chile (NMX-FF-108-SCFI-2007); el fruto se volteó constantemente con una pala limpia para homogenizar su deshidratado y ahumado, como se hace tradicionalmente.

Manipulación del trabajador. En los Tratamientos I, II y III no se permitió contacto físico del trabajador con el chile. Para ello se colocaron hileras de bloques que le permitieran pisar, acercarse y moverlo. En el Tratamiento IV el trabajador estuvo en contacto directo con el chile, es decir, lo pisó mientras lo volteaba. Se acondicionó un lavamanos rústico para que el obrero se lavara las manos antes de trabajar en los espacios de los Tratamientos I y II, y los instrumentos de trabajo estuvieron limpios.

Toma de muestras en el horno. Se tomaron muestras aleatorias de chile en cada tratamiento, en dos procesos efectuados durante noviembre del 2003. En cada fecha y en cada tratamiento se tomó una muestra diaria de chile equivalente a seis repeticiones por tratamiento. Cada 
muestra estuvo compuesta por $300 \mathrm{~g}$ de producto. Se tomaron 48 muestras durante los dos procesos, en las cuales se hizo análisis de coliformes fecales, E. coli y contaminación física. Las muestras de "Chipotle" se colectaron con guantes en bolsas de plástico estériles (Nasco WHIRL-PAK®) y se trasladaron al laboratorio en una hielera con geles refrigerantes (NOM-109-SSA11994).

\section{Experimento 2, Transporte}

Se evaluaron los puntos posibles de contaminación física y microbiológica durante el transporte del "Chipotle", desde el horno hasta la planta seleccionadora.

Superficies inertes. Se tomaron muestras de la plataforma de dos camiones donde se colocaría el fruto del "Chipotle" a granel. Para la toma de muestra de esta superficie se utilizaron hisopos estériles humedos con una solución amortiguadora de fosfatos, se frotaron sobre la plataforma del camión, y luego se introdujeron nuevamente en el tubo de ensayo que contenía la solución amortiguadora. Las muestras se sometieron a análisis de coliformes totales mediante la técnica de unidades formadoras de colonias, UFC (NOM-113-SSA1-1994).

Muestras de "Chipotle". Al azar se tomaron seis muestras de fruto de "Chipotle" que se transportaba a granel en los camiones. Las muestras se sometieron a análisis de contaminación física, coliformes fecales y $E$. coli.

\section{Experimento 3, Planta seleccionadora y almacén}

Superficies inertes. Se tomó una muestra del piso del almacén donde se colocó el fruto de chile antes de ser seleccionado. Para ello se aplicó la técnica del hisopo estéril y los tubos con solución amortiguadora de fosfatos. Las muestras se trasladaron al laboratorio (NOM-109SSA1-1994) para cuantificar las UFC de coliformes totales (NOM-113-SSA1-1994).

Superficies vivas. El diagnóstico de la higiene de los trabajadores de la planta seleccionadora se hizo mediante el análisis de coliformes totales, a partir de un muestreo tomado en la palma de las manos, entrenudos y uñas con un hisopo estéril. Se eligieron tres trabajadores que tenían contacto directo con el "Chipotle" en la planta seleccionadora.

Muestras de fruto. Se tomaron seis muestras de seis costales de "Chipolte". En estas muestras se determinó la contaminación física, coliformes fecales y $E$. coli. La contaminación física se determinó de manera visual.
Análisis microbiológicos. Para estos análisis se utilizaron las metodologías establecidas en la legislación mexicana (NOM-092-SSA1-1994; NOM-110-SSA1-1994; NOM-112-SSA1-1994; NOM-145-SSA1-1995).

Preparación de muestras. De cada muestra de fruto se tomaron tres submuestras de 10 g cada una, se trituraron en homogeneizador peristáltico (Stomacher Seward 400®) con $90 \mathrm{~mL}$ de solución amortiguadora de fosfato Butterfield (BFB) durante 1 min (NMX-F-308-1992), para la determinación de NMP de coliformes fecales (CF) y $E$. coli; y UFC para coliformes totales. A partir de cada homogeneizado se hicieron diluciones decimales 10, 1.0 (muestra directa), 0.1, 0.01 y 0.001. Para coliformes totales las diluciones fueron de 1.0, 0.1 y 0.01 (NOM-110SSA1-1994; Wallace y Geraldine, 1995).

Determinación de organismos coliformes totales y fecales. El número de organismos se estableció mediante la cuenta de unidades formadoras de colonias (UFC) con la técnica del número más probable (NMP). Esta última, también llamada técnica de dilución en tubo, proporciona una estimación estadística de la densidad microbiana con base en la probabilidad de obtener tubos de ensayo con crecimiento positivo. El método se basa en que las bacterias coliformes fermentan la lactosa al ser incubadas a $35 \pm 1{ }^{\circ} \mathrm{C}$ durante 24 a $48 \mathrm{~h}$ y producen ácidos y gas; este último se detecta en las campanas de fermentación tipo Durham (NOM-112-SSA1-1994), conforme a la metodología estándar del NMP por fermentación en tubos múltiples (NOM-112-SSA1-1994 y NMX-F-308-1992).

Se utilizaron tres series de diluciones de cinco tubos con caldo lauril sulfato (CLS), en los que se inocularon 10 $\mathrm{mL}$ (en lauril doble), 1, 0,1, 0,01 y $0.001 \mathrm{~mL}$ de cada muestra. Los tubos se incubaron a $35.5^{\circ} \mathrm{C}$ por $24 \mathrm{~h}$. De los tubos de CLS que presentaban crecimiento determinado por la presencia de turbidez y gas, se inoculó una asada en caldo EC y se incubó a $45^{\circ} \mathrm{C}$ por 24 h en baño maría. Al final de la incubación, se registraron las combinaciones de tubos positivos (formación de gas) y se determinó el $\mathrm{NMP} / \mathrm{g}$ de coliformes fecales para comparar los resultados obtenidos con los cuadros correspondientes en la NOM112-SSA1-1994.

Esta comparación se hizo de la siguiente manera: cuando sólo una dilución mostró tres tubos positivos, se eligió ésta y las diluciones mayores posteriores. Cuando más de una dilución mostró tres tubos positivos y la última tenía menos de tres, se eligió esta última y las dos diluciones anteriores más bajas (NOM-112-SSA1-1994). En cada caso se obtuvo un número de tres cifras, los cuales están representados en los cuadros de esta Norma 
junto a sus límites de confianza. En la columna que indica el número de tubos positivos se buscó el índice del NMP.

Para determinar coliformes totales, en cajas Petri con Agar Bilis y Rojo Violeta (RVBA) (Difco, USA) se colocó por duplicado $1 \mathrm{~mL}$ de la muestra directa y se hicieron dos diluciones $\left(10^{-1}\right.$ y $\left.10^{-2}\right)$. La muestra reposó en agar hasta solidificación; posteriormente se agregó $4 \mathrm{~mL}$ de RVBA a la superficie de la caja inoculada y se incubó a $35{ }^{\circ} \mathrm{C}$ durante $24 \mathrm{a} 48 \mathrm{~h}$. Se determinaron las $\mathrm{UFC} / \mathrm{cm}^{2}$ con el contador de colonias (Stuart Scientific $®$ ) (NOM-113SSA1-1994). El análisis se hizo por triplicado.

Determinación de $\boldsymbol{E}$. coli. Para su cuantificación se emplearon los tubos positivos a coliformes fecales y se hicieron dos pruebas del comportamiento metabólico de $E$. coli: fluorescencia y producción de indol. Para determinar fluorescencia se empleó caldo lactosado EC con 4metilumbelliferil- $\beta$-D-glucuronide (MUG) (Difco, Francia). Las muestras se incubaron a $35^{\circ} \mathrm{C}$ por $24 \mathrm{~h}$, y luego los tubos se expusieron a radiación UV (Lámpara UV-4BNF) a una longitud de onda de $365 \mathrm{~nm}$ para observar fluorescencia. Ésta indicó la existencia de la bacteria (Moberg, 1985; NOM-145-SSA1-1995). La producción de indol (FDA/CFSAN, 2001) se determinó mediante incubación en caldo triptosa y reactivo de Kovac (Sigma); si se producía una coloración rosada a fucsia, se consideraba como resultado positivo. En la prueba de fluorescencia el cálculo de $E$. coli por gramo de muestra se hizo con la metodología de los coliformes fecales. El valor obtenido se expresó como NMP/g de E. coli para comparar los resultados con los de la Norma. El NMP es un método estadístico que consiste en algunos pasos como el "presuntivo", "confirmativo" y "fase completa" (FDA/CFSAN, 2001 y NOM-145-SSA1-1995).

Uso de tablas de NMP con $95 \%$ de límite de confianza. Los cuadros de la NOM-145-SSA1-1995 aportan la estimación estadística de los valores del NMP que corresponden a $95 \%$ de límite de confianza cuando se utilizan 3, 5 y 10 tubos. El intervalo de $95 \%$ de confianza se interpretó de la siguiente manera: cuando el número observado de microorganismos estuvo dentro de los límites, se asumió que $95 \%$ de los eventos son correctos. El valor del NMP tabulado representa un intervalo y no un valor absoluto. El resultado de una determinación de cinco tubos donde tres de ellos resultaron positivos en $0.01 \mathrm{~g}$; dos tubos positivos en $0.001 \mathrm{~g}$ y uno positivo en $0.0001 \mathrm{~g}$ (3-2-1) se leyó como 17 en el Cuadro 2 de esta Norma, y se multiplicó por 10 para obtener 170 como el NMP actual por gramo de muestra. De igual forma, si la cantidad más grande utilizada para el cuadro de referencia fue $1 \mathrm{~g}$ en lugar de $0.1 \mathrm{~g}$, se dividió entre $10 \mathrm{NMP}$ derivado del cuadro. Por ejemplo, el resultado de la determinación del NMP en tres tubos para $E$ coli donde tres tubos fueron positivos en $1 \mathrm{~g}$, un tubo positivo en $0.1 \mathrm{~g}$ y ninguno

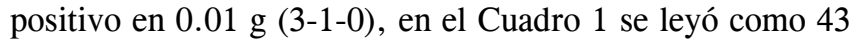
y se dividió entre 10 , lo que arrojó un valor de 4.3 como el NMP presuntivo por gramo de muestra.

Cuadro. 1. Descripción de tratamientos aplicados durante el proceso de producción del chile "Chipotle" en el horno de secado. Camargo, Chihuahua, Méx. 2003.

\begin{tabular}{llll}
\hline Tratamiento & \multicolumn{2}{c}{ Prácticas de manejo } \\
\hline I & $\begin{array}{l}\text { Superficie del horno lavada }{ }^{\dagger} \text { y } \\
\text { desinfestada } \\
\text { el fruto }\end{array}$ & $\begin{array}{l}\text { Horno cubierto con } \\
\text { malla }\end{array}$ & $\begin{array}{l}\text { Instrumentos de trabajo lavados. El obrero no tuvo contacto } \\
\text { con el fruto }\end{array}$ \\
II & $\begin{array}{l}\text { Superficie del horno sin lavar, pero } \\
\text { desinfestada }\end{array}$ & $\begin{array}{l}\text { Horno cubierto con } \\
\text { malla }\end{array}$ & $\begin{array}{l}\text { Instrumentos de trabajo lavados. El obrero no tuvo contacto } \\
\text { con el fruto }\end{array}$ \\
III & $\begin{array}{l}\text { Superficie del horno sin lavar pero } \\
\text { desinfestada }\end{array}$ & $\begin{array}{l}\text { Horno cubierto con } \\
\text { malla }\end{array}$ & $\begin{array}{l}\text { Instrumentos de trabajo sin lavar. El obrero no tuvo contacto } \\
\text { con el fruto }\end{array}$ \\
IV & $\begin{array}{l}\text { Superficie del horno sin lavar y sin } \\
\text { desinfestar }\end{array}$ & Sin cubrir & $\begin{array}{l}\text { Instrumentos de trabajo sin lavar. El obrero tuvo contacto } \\
\text { con el fruto }\end{array}$ \\
\hline F Lavado con agua, jabón y cepillo.
\end{tabular}




\section{RESULTADOS Y DISCUSIÓN}

Experimento 1, Horno chipotlero. En este experimento no se detectaron coliformes fecales en los tratamientos en donde los frutos se cubrieron con mallasombra, se utilizaron instrumentos de trabajo previamente lavados y se evitó el contacto físico de los obreros con el fruto de chile (Tratamientos I y II). En las muestras del Tratamiento III se encontró un promedio de $93 \mathrm{NMP} / \mathrm{g}$ de coliformes fecales; en este tratamiento, a diferencia de los anteriores, no se lavaron los instrumentos de trabajo. El fruto del Tratamiento IV fue el más contaminado, con un promedio de $210 \mathrm{NMP} / \mathrm{g}$ debido a que no se aplicaron buenas prácticas de manejo (Cuadro 2).

De las 48 muestras de "Chipotle" analizadas, $50 \%$ fueron positivas para coliformes fecales, y todas las muestras positivas fueron tomadas de los tratamientos en donde no se aplicaron BPM. Por otra parte, las 48 muestras mostraron ausencia de E. coli (Cuadro 2), tal vez debido a que algunos constituyentes del humo de madera tienen una función antimicrobiana. Entre estos compuestos se encuentran los carbonílicos como el formaldehido, los fenólicos como el guaiacol, y los ácidos como fórmico y acético (Ogbadu, 2004; Serot, 2004). Al colocar los bloques para evitar que el trabajador entrara en contacto con el chile al moverlo, se logró disminuir considerablemente la contaminación microbiológica por coliformes fecales.

La malla antigranizo detuvo la entrada de insectos y roedores al horno. Fuera de la malla, alrededor del horno se observaron algunos contaminantes físicos como plumas y excremento de aves. La malla detuvo la contaminación que pudo ser provocada por hojas, ramas y bolsas de plástico acarreados por el viento hacia los hornos.

Cuadro 2. Contaminación física total, unidades formadoras de colonias (UFC $\left./ \mathrm{cm}^{2}\right)$ de coliformes totales (CT) y número más probable/g de coliformes (NMP) fecales y $E$. coli en chile "Chipotle" y en superficies vivas e inertes en tres experimentos. Camargo, Chihuahua, Méx. 2003.

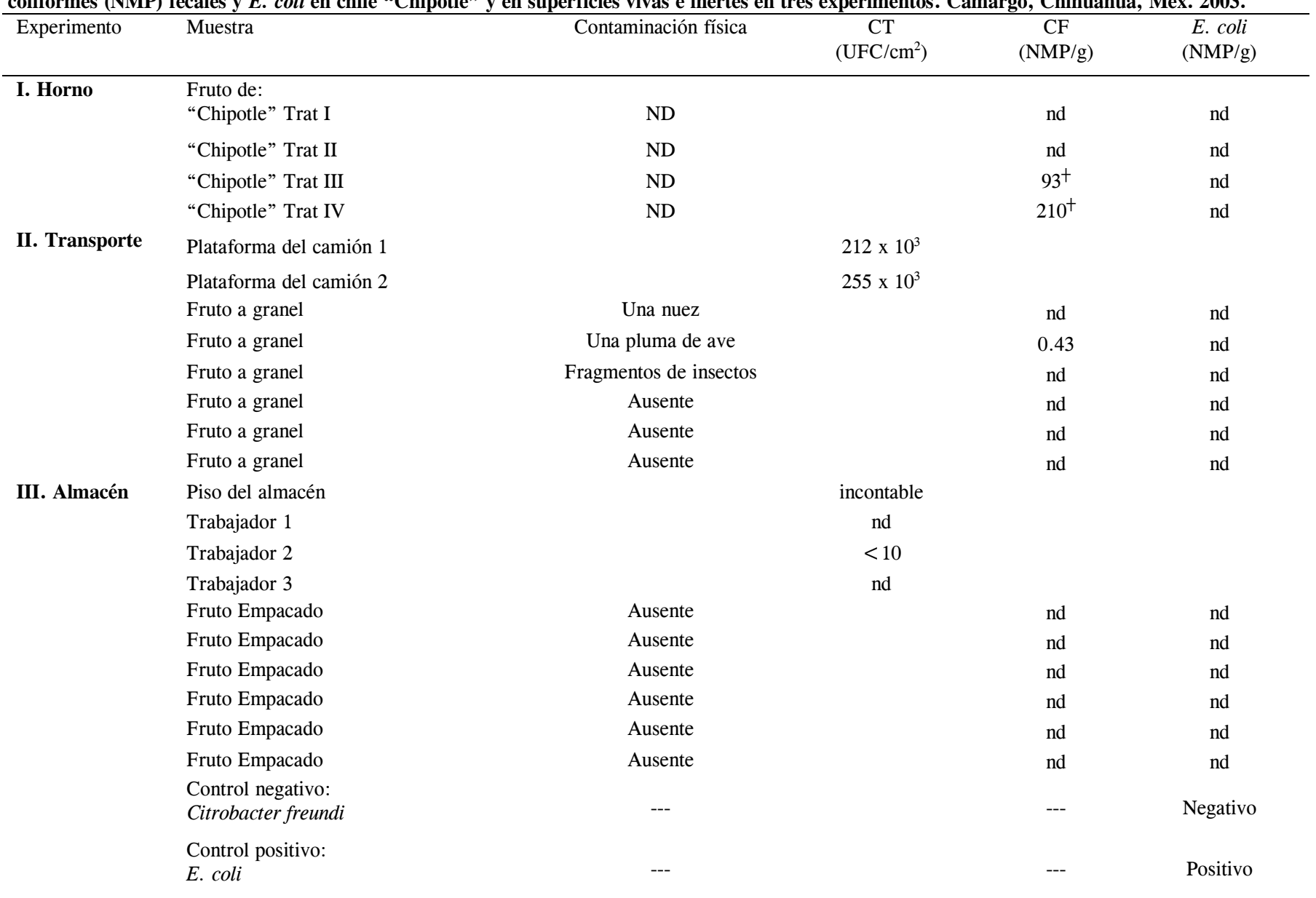

nd $=$ No detectable; CT $=$ Organismos coliformes totales; CF $=$ Organismos coliformes fecales; UFC $=$ Unidades formadoras de colonias; NMP $=$ Número más probable; ${ }^{\dagger}$ Promedios $(\mathrm{u}=6)$. El experimento 1 se realizó dos veces. 
Experimento 2, Transporte. De seis muestras tomadas de frutos de "Chipotle" transportado a granel en camiones, tres presentaron contaminación física. Las muestras con contaminación física contenían una nuez pecanera, una pluma de ave y fragmentos de insecto (Cuadro 2). Este nivel de contaminación estuvo dentro de los límites indicados en la NMX-FF-108-SCFI-2007 para chile "Chipotle" donde especifica un límite máximo de 50 fragmentos de insecto en $25 \mathrm{~g}$ y menos de $1 \%$ de otro tipo de contaminación. La muestra de chipotle tomada del transporte que contenía una pluma de ave también presentó contaminación por coliformes fecales $(0.43 \mathrm{NMP} / \mathrm{g})$, tal vez debido al acarreo de material fecal por las aves. Muchas evidencias muestran asociación de coliformes con aves, y también se ha documentado que las bacterias pueden ser transportados por agua, viento o animales (Bopp et al., 2003; Nicholas, 2005; Singh et al., 2006). Los microorganismos patógenos pueden encontrarse en el aire aunque no se multiplican en él; en este ambiente su periodo de vida es corto por causa de la desecación, el ozono y la radiación ultravioleta.

Siempre se ha considerado a $E$. coli como indicador de contaminación fecal por ser una bacteria abundante en el intestino $\mathrm{y}$, como consecuencia, se encuentra en heces humanas y animales, por lo que su búsqueda en alimentos o agua genera un indicativo de contaminación fecal reciente (Bopp et al., 2003; Mukherjee et al., 2006). Aunque la mayoría de cepas de $E$. coli no son patogénicas, algunas cepas como E. coli 0157:H7 poseen una dosis mínima infectante muy baja (10-100 células) (Castro-Rosas et al., 2006). E. coli no se detectó en las muestras de "Chipotle" analizadas en este estudio mediante la prueba de fluorescencia (Moberg, 1985). Los coliformes totales estuvieron ausentes, excepto en la muestra del costal 2 con $0.43 \mathrm{NMP} / \mathrm{g}$.

Las superficies inertes del transporte mostraron la mayor contaminación con coliformes totales, con valores desde $212 \times 10^{3} \mathrm{UFC} / \mathrm{cm}^{2}$ o superiores. Esto mostró falta de limpieza de los camiones donde se transporta el producto agrícola.

Experimento 3, Seleccionadora y almacén. Las manos de las personas que seleccionaron el chile estaban prácticamente limpias, de modo que la baja contaminación por coliformes totales $(<10)$ en las manos del único trabajador que fue positivo no representó peligro (Cuadro 2). No se indicó a los obreros que se lavaran las manos antes del muestreo. Después de haber sido seleccionado y empacado el fruto no presentó contaminación física ni microbiológica. En este trabajo la mayor contaminación se observó en las superficies inertes, según el conteo de coliformes totales en el piso de almacén con UFC incontables. Es necesario mencionar que estos análisis se realizaron antes de colocar el fruto en el almacén, cuya superficie se lavó antes de almacenar el "Chipotle". Estos resultados mostraron como se puede prevenir la contaminación cruzada mediante BPM.

No resulta fácil establecer el nivel de riesgo asociado con cierta concentración de coliformes fecales o totales. Sin embargo, estos resultados muestran el efecto de no aplicar BPM (FAO, 2008; SAGARPA, 2008). Los resultados de este estudio demuestran la importancia de lavar las superficies de contacto, de colocar una malla alrededor del horno para evitar la entrada de roedores o aves, de lavar los utensilios que entran en contacto con el producto y de minimizar el contacto del trabajador con el producto. Al aplicar medidas preventivas de higiene es posible obtener un producto limpio, aun cuando el proceso se realice en campo abierto. Las prácticas que se hicieron en el horno requirieron una baja inversión y aseguraron la calidad microbiológica del "Chipotle", confirmada con la baja contaminación con coliformes fecales y la ausencia de E. coli.

\section{CONCLUSIONES}

La contaminación física y microbiológica del chile "Chipotle" se atribuye a la manipulación por el hombre y a la falta de aislamiento del producto.

El "Chipotle" tratado con buenas prácticas de manejo no mostró contaminación por coliformes fecales, porque el trabajador de campo cumplió cabalmente estas prácticas. El recubrimiento del horno con malla evitó la introducción de roedores, insectos y materia extraña al chipotle. Se corroboró que la carga microbiana en el "Chipotle" es baja, aun cuando el proceso sea al aire libre.

\section{AGRADECIMIENTOS}

A los financiadores de este proyecto: la Secretaría de Desarrollo Industrial del Gobierno del Estado de Chihuahua, a través del INADET, y al empresario Chihuahuense, Ing. Humberto Márquez Pizarro. A la IQAl Hilda Sáenz Hidalgo e IQAl Alexandro Guevara Aguilar por el apoyo en la toma de datos.

\section{BIBLIOGRAFÍA}

Bopp D J, D B Sauders, A L Waring, J Ackelsberg, N Dumas, E Braun-Howland, D Dziewulski, B J Wallace, M Kelly, T Halse, K A Musser, P F Smith, D L Morse, R J Limberger (2003) Detection, isolation, and molecular subtyping of Escherichia coli $\mathrm{O} 157: \mathrm{H} 7$ and Campylobacter jejuni associated 
with a large waterborne outbreak. J. Clinical Microbiol. 41:174180.

Castro-Rosas J, M Rojas-Olvera, Y Noguera-Ugalde, E M SantosLópez, A Zúniga-Estrada, C A Gómez-Aldapa (2006) Calidad sanitaria de ensaladas de verduras crudas, listas para su consumo. Rev. Alfa Eds. Téc. Julio-Agosto: 9-21.

Doyle M E, J Archer, C W Kaspar, R Weiss (2006) Human illness caused by $E$. coli $0157: \mathrm{H} 7$ from non-food sources. FRI Briefings: E. coli 0157:H7 Human illness from food and nonfood sources. Food Research Institute, University of Wisconsin, Madisson. USA. 37 p. Disponible en: www.wisc.edu/fri/ briefs/FRIBrief_EcoliO157H7humanillness.pdf (Enero, 2009).

Fairbrother J M, E Nadeau (2006) Escherichia coli: on-farm contamination of animals. Rev. Sci. Tech. 25:555-569.

FAO (2008) Food Agricultural Practices. FAO Key Documents on Good Agricultural Practices (GAP). Disponible en: www.fao.org/ prods/gap/index_en.htm (Enero 2009).

FDA/CFSAN (2001) Bacteriological Analytical Manual Online. Disponible en: www.cfsan.fda.gov/\%7Eebam/bam-4.html (Enero 2009). Equivalente a Bacteriological Analytical Manual, 1988, 8th ed. Chapter 4. AOAC Internatl. USA.

Hilborn E D, J H Mermin, P A Mshar, J L Hadler, A Voetsch, C Wojtkunski, M Swartz, R Mshar, F M Lambert, J A Farrar, M K Glynn, L Slutsker (1999) A multistate outbreak of Escherichia coli $0157: \mathrm{H} 7$ infections associated with consumption of mesclun lettuce. Arch. Internatl. Med. 159:1758-1764.

Jiménez D F, M Y Chew (2002) Practicas para producción inocua de melón. In: El Melón: Tecnologías de Producción y Comercialización. J J Espinoza (ed). INIFAP, Libro técnico No. 4. México. pp:219-242.

Leclercq A, C Wanegue, P Baylac (2002) Comparison of fecal coliform agar and violet red bile lactose agar for fecal coliform enumeration in foods. Appl. Environ. Microbiol. 68:1631-1638.

Martínez A, W Tejedor-Espinosa, M P Cano, B de Ancos, P S Fernández (2005) Control de etapas críticas en el procesamiento de productos cortados (HACCP). In: Nuevas Tecnologías de Conservación de Productos Vegetales Frescos Cortados. G González, A A Gardea, F Cuamea (eds). Ed. Logiprint Digital S. de R.L. de C.V. Jalisco, México. pp:501-520.

Moberg L J (1985) Fluorogenic assay for rapid detection of Escherichia coli in food. Appl. Environ. Microbiol. 50:1383-1387.

Mukherjee A, S Cho, J Scheftel, S Jawahir, K Smith, F DiezGonzalez (2006) Soil survival of Escherichia coli 0157:H7 acquired by a child from garden soil recently fertilized with cattle manure. J. Appl. Microbiol. 101:429-436.

Nicholas G L (2005) Fly transmission of Campilobacter. Emerg. Infec. Dis. 11:361-364.

Norma Mexicana NMX-FF-108-SCFI-2007 Productos Alimenticios Chile Chipotle o Chilpotle (Capsicum annuиm) Especificaciones y Métodos de Prueba. SAGARPA.
Norma Mexicana NMX-F-308-1992 Alimentos. Cuenta de Microorganismos Coliformes Fecales. Foods - Fecals Coliform Organisms Count. Dirección General de Normas.

Norma Oficial Mexicana NOM-092-SSA1-1994 Bienes y Servicios. Método para la Cuenta de Bacterias Aerobias en Placa. Secretaria de Salud.

Norma Oficial Mexicana NOM-109-SSA1-1994 Bienes y Servicios. Procedimiento para la Toma, Manejo y Transporte de Muestras de Alimentos para su Análisis Microbiológico. Secretaria de Salud.

Norma Oficial Mexicana NOM-110-SSA1-1994 Bienes y Servicios. Preparación y Dilución de Muestras de Alimentos para su Análisis Microbiológico. Secretaria de Salud.

Norma Oficial Mexicana NOM-112-SSA1-1994 Bienes y Servicios. Determinación de Bacterias Coliformes. Técnica del Número más Probable. Secretaria de Salud.

Norma Oficial Mexicana NOM-113-SSA1-1994 Bienes y Servicios. Método para la Cuenta de Microorganismos Coliformes Totales en Placa. Secretaria de Salud.

Norma Oficial Mexicana NOM-145-SSA1-1995 Productos Cárnicos Troceados y Curados. Productos Cárnicos Troceados y Madurados. Disposiciones y Especificaciones Sanitarias. Secretaria de Salud.

Ogbadu L J (2004) Preservatives, traditional preservatives, wood smoke. In: Encyclopedia of Food Microbiology. R K Robinson, C A Batt, P Patel (eds). Cornell University, Department of Food Science, Ithaca, New York, USA. pp:1737-1743.

Pouch F D, K Ito (2001) Compendium of Methods for the Microbiological Examinatiopn of Food. American Public Healt Association. Washington DC, USA. 4th ed. $676 \mathrm{p}$.

SAGARPA (2008) Inocuidad Agrícola. Buenas Prácticas Agrícolas. Lineamientos. Disponible en: www.senasica.gob.mx (Enero 2009).

Serot T (2004) Effect of smoking processes on the contents of 10 major phenolic compounds in smoked fillets of herring (Cuplea harengus). Food Chem. 85:111-120.

SIAP (2008) Servicio de Información Agroalimentaria y Pesquera. Disponible en: www.siap.gob.mx (Enero 2009).

Singh B R, P Singh, A Verma, S Agarwal, N Babu, M Chandra, R K Agarwal (2006) A study of prevalence of multi-drug-resistant (MDR) Salmonella in water sprinkled on fresh vegetables in Bareilly, Moradabad and Kanpur (northern Indian cities). J. Public Health 14:125-131.

Söderström A, A Lindberg, Y Andersson (2005) EHEC 0157 outbreak in Sweden from locally produced lettuce. Euro Surveill 10: 2794. Disponible en: www.eurosurveillznce org/View Article.aspx? Articleld = 2794 (Enero 2009).

Vlkova E, I Trojanova, V Rada (2006) Distribution of bifidobacteria in the gastrointestinal tract of calves. Folia Microbiol. 51:325-328.

Wallace H A, A J Geraldine (1995) Food and Drug Administration. Bacteriological Analytical Manual. AOAC. Chapter 1, 8th ed. Bethesda: AOAC International, 1.01-1.09, USA. 